\title{
Elisabeth J. Moodey, Illuminated Crusader Histories for Philip the Good of Burgundy
}

\section{Maria Colombo Timelli}

\section{(2) OpenEdition}

1 Journals

\section{Édition électronique}

URL : http://journals.openedition.org/studifrancesi/354

DOI : 10.4000/studifrancesi.354

ISSN : 2421-5856

Éditeur

Rosenberg \& Sellier

\section{Édition imprimée}

Date de publication : 1 avril 2015

Pagination : 128-130

ISSN : 0039-2944

\section{Référence électronique}

Maria Colombo Timelli, «Elisabeth J. Moodey, Illuminated Crusader Histories for Philip the Good of Burgundy », Studi Francesi [En ligne], 175 (LIX | I) | 2015, mis en ligne le 01 avril 2015, consulté le 18 septembre 2020. URL : http://journals.openedition.org/studifrancesi/354 ; DOI : https://doi.org/ 10.4000/studifrancesi.354

Ce document a été généré automatiquement le 18 septembre 2020.

\section{(c)}

Studi Francesi è distribuita con Licenza Creative Commons Attribuzione - Non commerciale - Non opere derivate 4.0 Internazionale. 


\title{
Elisabeth J. Moodey, Illuminated Crusader Histories for Philip the Good of Burgundy
}

\author{
Maria Colombo Timelli
}

\section{RÉFÉRENCE}

ELISABETH J. MOODEY, Illuminated Crusader Histories for Philip the Good of Burgundy, Turnhout, Brepols, 2012 ( «Ars Nova, Studies in Late Medieval and Renaissance Northern Painting and Illumination»), pp. 312.

1 Ce bel ouvrage, imprimé sur papier glacé et richement illustré, intéressera les spécialistes de plusieurs domaines: historiens de l'art, du livre, de la littérature et de la politique bouguignonnes. Il concerne en effet un des aspects les mieux connus et les plus cités - parfois avec quelque condescendence - de la politique extérieure de Philippe le Bon: ce «rêve», et le mot n'est pas anodin, d'organiser une croisade qui l'a accompagné tout au long de sa vie, avant et après la chute de Constantinople dans les mains des Turcs en 1453. Disons tout de suite que la perspective de E.J. Moodey est celle d'une historienne de l'art, et que par conséquent il serait injuste de prétendre de sa part une égale connaissance dans les différents domaines qu'elle s'efforce d'aborder; on verra cependant que les questions proprement littéraires auraient mérité un approfondissement ultérieur.

2 Après l'Introduction (pp. 1-17), consacrée à une bonne synthèse sur le patronage artistique de Philippe le Bon en rapport avec la Croisade, et en particulier sur les textes et les manuscrits qu'il a commandités, reçus ou fait illustrer, le livre est organisés en cinq chapitres.

3 Les deux premiers concernent la production bourguignonne sur l'Histoire et en particulier l'histoire des Croisades: fort opportunément E.J. Moodey signale que la partition entre chroniques et romans historiques n'est que conventionnelle (p. 15). 
Dans le chapitre I (The Literature of Fact: History Writing at the Burgundian Court, pp. 19-51), un rapide aperçu de l'écriture historique médiévale introduit les œuvres plus centrées sur les territoires bourguignons et dont Philippe le Bon possédait une ou plusieurs copies: L'estore des contes de Flandres, Chronique des ducs de Brabant (version latine d'Edmond de Dynter et traduction française de Jean Wauquelin), Chroniques de Hainaut (version latine de Jacques de Guise et traduction du même Wauquelin), Chronique de Hollande, de Zeelande et de Frise. Une dernière section est consacrée à Georges Chastelain et à l'importance de sa charge d'historiographe officiel du Duc. Le deuxième chapitre (The Literature of Fiction: Historical Romances and 'mises en prose', pp. 53-78) est intéressant dans la mesure où il fournit une vision d'ensemble de la littérature de fiction où le thème de la Croisade représente un noyau fondamental du récit; les spécialistes de littérature bourguignonne n'y feront pas de découvertes, à plus forte raison que la bibliographie utilisée est souvent périmée voire incomplète. On regrette surtout que les textes soient cités sous des intitulés anciens et parfois méconnaissables (pourquoi avoir adopté Istoire royale, «sometimes known as Roman de trois fils de Roy or Chroniques de Naples», p. 76, plutôt que Les trois fils de rois, selon l'édition de Giovanni Palumbo, Paris, H. Champion, 2002, pourtant citée? Et, pire, pourquoi avoir inventé le titre L'Ystoire de Helayne, Mère de Saint Martin, evesque de Tours pour la mise en prose de Jean Wauquelin, titre qui ne se lit même pas dans le manuscrit Bruxelles, KBR 9967, alors que La Belle Hélène de Constantinople est le titre couramment adopté?) et que les éditions critiques parues ces dernières années soient tout à fait ignorées: pour me limiter à ce dernier exemple, l'édition par Marie-Claude de Crécy, pourtant publiée il y a douze ans et dans une collection diffusée dans toutes les bibliothèques (Genève, Droz, 2002, «TLF» 547), n'est citée ni dans le texte ni dans la bibliographie finale; les citations du prologue sont tirées de la monographie de Richard Straub (David Aubert, 'escripvain' et 'clerc', Amsterdam, Atlanta, Rodopi, 1995), importante certes, mais dépassée pour ce qui concerne justement l'édition des textes. Je signale en outre que les études sur l'iconographie du manuscrit unique de cette mise en prose, illustré par Loyset Liédet, abondent et ne devraient pas être inconnues à une historienne de l'art (voir à ce propos la notice Belle Hélène de Constantinople sur le site http://users2.unimi.it/lavieenproses/). Sans multiplier les exemples, signalons au moins que Le Châtelain de Coucy est maintenant édité (par Aimé Petit et François Suard, Villeneuve d'Ascq, Presses Universitaires du Septentrion, 1994), ainsi que Gilles de Chin (et non pas Gilles du Chin: p. 72; éd. par Anne-Marie Liétard-Rouzé, même éditeur, 2010): les deux éditions contiennent la reproduction - la première sur papier, la seconde sur CD - des enluminures dues au 'Maître de Wavrin'. Tous ces textes sont cités de seconde main à partir de l'ouvrage de Georges Doutrepont (1939).

4 Trois autres chapitres concernent spécialement l'histoire des Croisades. Plus en particulier, dans Crusader Past (chap. III, pp. 78-123), E.J. Moodey parcourt la suite de ces guerres entre le $\mathrm{xI}^{\mathrm{e}}$ siècle et 1453 , en soulignant la participation des ancêtres de Philippe le Bon et le reflet de ces évènements, parfois tragiques pour les Valois (ainsi, l'emprisonnement de Jean sans Peur à Nicopolis, le jour même de la naissance de Philippe), dans la «librairie» ducale. Les œuvres prises en compte vont du Livre d'Éracles aux Récits d'un Ménestrel de Reims, de la Chronique de Baudouin d'Avesnes à l'Histoire de saint Louis de Joinville, de la Fleur des Histoires de la Terre d'Orient de Hayton au Liber secretorum de Marino Sanudo; entre la fin du XIV et le $\mathrm{xv}^{\mathrm{e}}$ siècle les textes se multiplient et se différencient: l'Epistre lamentable de Philippe de Mézières côtoie sur les rayons de Bourgogne les rapports de voyage de Guillebert de Lannoy ou le Voyage d'Oultremer de 
Bertrandon de la Brocquière, mais aussi le Desbat du Chrestien et du Sarrazin de Jean Germain. Sont aussi rappelés quelques évènements marquants du règne de Philippe le Bon qui eurent des retombées profondes sur ses collections: le mariage avec Isabelle du Portugal en 1430 et, la même année, la création de l'ordre chevaleresque de la Toison d'or. Un chapitre à part est bien entendu consacré à The Fall of Constantinople and the Banquet of the Pheasant (chap. IV, pp. 125-173); la synthèse offerte par E.J. Moodey s'appuie sur la bibliographie existante, très abondante: on pourra y ajouter, pour ce qui concerne l'habitude de porter du noir à la cour de Bourgogne, le bel article de Sophie Jolivet, La construction d'une image: Philippe le Bon et le noir (1419-1467) (in Se vêtir à la cour en Europe, 1400-1815, Université de Lille 3 - Charles de Gaulle, 2011, pp. 27-42). Le chapitre Crusades Present (V, pp. 149-173) reprend et approfondit l'analyse de quelques œuvres et manuscrits spécialement consacrés au renouvellement du projet de Croisade après la chute de Constantinople: le Voyage de Bertrandon de la Brocquière (conservé dans le ms KBR 9087 avec deux traductions de Jean Miélot: l'Advis directif pour faire le voyage d'Oultremer et la Description de la Terre Sainte, 1455), la «lettre» de Jacopo Tedaldi (ms BnF, fr. 6487; sur ce texte il faudra renvoyer à l'importante contribution de Monica Barsi, Constantinople à la cour de Philippe le Bon, 1419-1467. Compte rendus et documents historiques, avec l'édition du manuscrit BnF fr. 2691 du récit de Jacopo Tedaldi, in Sauver Byzance de la Barbarie du monde, Milano, Cisalpino, 2004, pp. 131-195), la Lamentation de Grèce de Jean Molinet (1464), et l'anonyme Epistre en la contemplacion du saint voyage de Turquie (1464).

5 Deux chapitres monographiques sont enfin consacrés à deux manuscrits commandités par Philippe le Bon: les Croniques de Jherusalem (Wien, ÖNB, 2533) et les Chroniques et conquestes de Charlemaine (Bruxelles, KBR, 9066-68). Les reproductions en couleur des pages et enluminures objet de commentaire permettent de suivre au mieux le discours de E.J. Moodey et d'apprécier son interprétation des programmes iconographiques et de la mise en page de ces beaux livres; il en est ainsi en particulier pour le manuscrit de Vienne (chap. VI, Godfrey of Bouillon and "Les croniques de Jherusalem abregies", pp. 175-208), dont la mise en page horizontale - similaire à celle que Jean Miélot adopta pour ses Histoires de toute la Bible, ms KBR II-239 - permet de mettre en relief le déroulement de l'histoire, entre autres en proposant des arbres généalogiques parallèles. Le célèbre manuscrit KBR 9066-68, fait l'objet du dernier chapitre (Charlemagne et «Les cronicques et conquestes de Charlemaine», pp. 209-239): copié par David Aubert et décoré par de somptueuses grisailles dues au moins en partie à Jean le Tavernier, ce codex constitue un véritable monument de l'histoire du livre bourguignon. Les analyses des enluminures permettent même à un lecteur peu averti d'apprécier la technique mise en œuvre par l'illustrateur, les détails, la finesse du trait, la mise en relief de quelques personnages ou de leurs gestes. Une telle commande - il est douteux si c'est Philippe le Bon ou Jean de Créquy qui prit l'initiative - s'explique nécessairement par un profond intérêt de la part du Duc pour l'histoire de l'empereur dans lequel il voyait un de ses ancêtres. Dans ce cas encore, je me permets de signaler une contribution toute récente qui porte sur ce même manuscrit et notamment sur le rapport entre texte et enluminures: Martine Thiry-Stassins, La parole racontée et l'histoire montrée: David Aubert et Jean Le Tavernier ("Croniques et Conquestes de Charlemaine», ms. KBR 9068), in Pour un nouveau répertoire des mises en prose. Romans, chansons de geste, autres genres, Paris, Classiques Garnier, 2014, pp. 321-345.

6 La Conclusion (pp. 241-245) met d'abord en relief le triple déplacement de Paris vers la Bourgogne qui se reconnaît entre la fin $d u x^{2} v^{e}$ et la fin $d u x^{e}$ siècle: art du livre 
manuscrit, production littéraire et écriture de l'Histoire trouvent un milieu fertile et de nouveaux patrons et mécènes sous les ducs de Valois et à leur cour. Cette identité culturelle à part s'exprime entre autres dans les projets de Croisade et dans la représentation réservée dans certains manuscrits à des héros tels que Charlemagne ou Godefroy de Bouillon, protagonistes de la lutte contre les infidèles et ancêtres prétendus de Philippe le Bon. C'est dans cette même optique de «propagande» ducale qu'il faudra resituer la charge d'indiciaire assumée par Georges Chastelain ou l'évènement politique que fut le Banquet du faisan, ainsi que l'énorme production de manuscrits luxueux commandités par le Duc et ses proches.

7 Quelques compléments enrichissent ultérieurement ce beau volume: une transcription des Croniques de Jherusalem abregies (pp. 247-259), une biographie de Jean le Tavernier (pp. 261-266), la bibliographie (sources primaires, pp. 267-269, et secondaires, pp. 269-283), index des manuscrits (pp. 285-287) et index général (titres, auteurs, sujets: pp. 289-311). 